\title{
ARtículos
}

\section{La Semana del Migrante en la Utec}

\section{Migrants' Week at UTEC}

\author{
Elsa Ramos \\ Investigadora Utec \\ elsa.ramos@utec.edu.sv \\ Recibido: 05/11/2015 - Aceptado: 24/11/2015
}

\section{Resumen}

La Universidad Tecnológica de El Salvador, Utec, tiene ya diez años consecutivos de realizar en su campus la Semana del Migrante, espacio para el análisis y discusión del fenómeno migratorio. En este espacio se han desarrollado múltiples temas desde diversas disciplinas, todos con la intención de buscar la mejor forma de tratar esta problemática. En la Utec se han alcanzado a más de 10 mil oyentes en las conferencias, foros, charlas, conversatorios y demás actividades que se han desarrollado.

\section{Palabras clave}

Migración, fenómeno migratorio, academia.

La migración es tan antigua como la humanidad misma. Desde la antropología se afirma que el desarrollo de las civilizaciones no hubiera sido posible sin la migración. Actualmente, y de acuerdo con los datos proporcionados por la ONU (2013) y la Organización para la Cooperación y el Desarrollo Económicos (OECD, por sus siglas en inglés) (2013), había en el mundo 232 millones de migrantes internacionales.

El Salvador no es ajeno al fenómeno social de las migraciones desde hace mucho tiempo, pero no es sino hasta finales de la década de los 70 del siglo XX cuando

\section{Abstract}

Universidad Tecnológica de El Salvador, UTEC, has conducted the Migrants' Week for ten consecutive years at its campus. During this activity, a space for the analysis and discussion of migration has been created. Multiple topics from diverse disciplines have been developed so far; all of them intend [to offer] the best way in which to solve the issue of migration. Until today, UTEC has reached more than 10,000 attendees during the lectures, forums, talks, discussions, and all other related activities within this event.

\section{Keywords}

Migration, migratory phenomenon, academia.

se visualiza una masiva ola migratoria de su población. Así, de acuerdo con el mapa de las migraciones salvadoreñas (2011), son 2.950 .126 las personas migrantes distribuidas en varios países del mundo; pero la nación que presenta mayor índice de recepción de migrantes es Estados Unidos de Norteamérica, ascendiendo a 2.587.767 los migrantes de origen salvadoreño que viven allá.

La Utec inicia su trabajo de investigación permanente en la línea de migraciones de forma continua a partir del año 2005 con la colaboración que se realizó para elaborar el capítulo

1 Licenciada en Historia, con una maestría en Ciencias Históricas por la Universidad Estatal del Kuban, Rusia. Candidata a Maestra en Estudios Latinoamericanos por la Universidad Nacional Autónoma de México, UNAM. Investigadora a tiempo completo de la Universidad Tecnológica de El Salvador, Utec. 
I del "Informe de Desarrollo Humano 2005. Una mirada al nuevo nosotros". Gracias a esa colaboración, se toma conciencia de que el fenómeno migratorio transversaliza a la sociedad salvadoreña en su conjunto.

A partir del año 2006 la Utec inicia la celebración de la Semana del Migrante, considerando que el fenómeno social de las migraciones es un tema de nación. Por ello es importante dar a conocer dicho fenómeno, ante la necesidad de sensibilizar y concientizar a la población estudiantil propia y a la sociedad en su conjunto de los riesgos y beneficios de dicho fenómeno. También se toma como apoyo legal el Decreto $\mathrm{N}^{\circ} 71$, acordado por la Asamblea Legislativa en el año de 1997, y cuyo artículo 1 reza de la siguiente forma:

Art. 1.- Declárase la primera semana del mes de septiembre de cada año "SEMANA DEL MIGRANTE", como un reconocimiento al gesto humanitario que realizan los ciudadanos salvadoreños que se encuentran en el extranjero en las diferentes partes del mundo.
Los objetivos principales de la celebración de la "Semana del migrante" en la Utec son abrir un espacio en donde se presenten las diferentes ópticas del fenómeno migratorio, en donde haya cabida para todos los sectores que están trabajando el tema, sin exclusiones de ningún tipo, pues lo importante es que se visualice el tema lo más ampliamente posible, que se descubran o pongan sobre la mesa los problemas claves que necesitan una solución inmediata, buscar entendimientos, acuerdos, para solventar dichos problemas, como por ejemplo, el aumento masivo de la migración de niños y niñas acompañados, no acompañados y separados, el aumento ya visible de mujeres, la migración forzada por violencia social, los desplazamientos internos forzados, igual, por la violencia social generada por los grupos que forman las maras.

Así, la Utec, hasta el año 2015, ha celebrado con muchos logros durante una década la Semana del Migrante. A continuación se presenta una sistematización de lo realizado en ese período, que incluye instituciones, representantes, temas que se han abordado y las actividades paralelas a dicha celebración.

\section{Instituciones invitadas a la Semana del Migrante}

\begin{tabular}{|l|l|l|l|}
\hline \multicolumn{1}{|c|}{ Instituciones gubernamentales } & \multicolumn{1}{|c|}{$\begin{array}{c}\text { Organismos } \\
\text { internacionales }\end{array}$} & \multicolumn{1}{|c|}{$\begin{array}{c}\text { Instituciones } \\
\text { educativas }\end{array}$} & ONG \\
\hline $\begin{array}{l}\text { Viceministerio para los Salvadoreños en el } \\
\text { Exterior }\end{array}$ & OIM & Upan & Cofamide \\
\hline Dirección General de Migración y Extranjería & Acnur & UCA & Funde \\
\hline Ministerio de Trabajo & laes/Pares & UES & Adel Morazán \\
\hline $\begin{array}{l}\text { Consejo Nacional para la Niñez y la } \\
\text { Adolescencia }\end{array}$ & Unicef & Universiad de Zacatecas & Confres \\
\hline Instituto Nacional para la Juventud & UNFPA & $\begin{array}{l}\text { Pontificia Universidad } \\
\text { Católica de Perú }\end{array}$ & Redmigres \\
\hline Consulado de Acayucan & Ciesas & CDHES \\
\hline $\begin{array}{l}\text { Comisión de Exteriores de la Asamblea } \\
\text { Legislativa }\end{array}$ & ICMD/PNUD & & Orden San Carlos Scalabrini \\
\hline PDDH & PNUD & & Menamig \\
\hline BCR & FIDH & & Asefin \\
\hline Consulado de Tapachula & ICMD/PNUD & & Save the Children \\
\hline PNC División de Fronteras & Unicef & & GMIES \\
\hline Conna & Visión Mundial & & Insami \\
\hline MJySP & & & Arpas \\
\hline
\end{tabular}




\begin{tabular}{|l|l|l|l|}
\hline Comité Nacional contra la Trata de Personas & & & Uscri \\
\hline Secultura & & & $\begin{array}{l}\text { Carecen Internacional - } \\
\text { El Salvador }\end{array}$ \\
\hline Alcaldía municipal de San Salvador & & & CRS \\
\hline Banco Hipotecario & & & Alpimed \\
\hline Ministerio de Trabajo & & & Seem \\
\hline & & & Redes \\
\hline & & & Cáritas El Salvador \\
\hline & & & Confres/JyP \\
\hline & & & Ucrica \\
\hline SANN & & & \\
\hline
\end{tabular}

Otras organizaciones: Fedecaces, Grupo de teatro y danza de la Casa de la Juventud Ángel de la Guarda.

Fuente: Cuadro propio realizado a partir de los programas de la Semana del Migrante realizada en la Utec.

\section{Personas invitadas}

\begin{tabular}{|l|l|}
\hline Institución & Personas invitadas \\
\hline & Juan José García \\
& Liduvina Magarín \\
& Davis Ernesto Morales Cruz \\
& José Manuel Castillo \\
& Doris Rivas \\
& Ernesto Nosthas \\
& Luis Perdomo Vidal \\
& Antonio Enrique Azúcar Hernández \\
& Vitelio Sánchez \\
& Roberto Turcios \\
& Helen Flamenco \\
& Ana Solórzano \\
Sandra Lovo \\
Karina Sosa \\
Carlos Acevedo \\
& Óscar Ovidio Cabrera Melgar \\
& José Noé Ayala \\
& Eunice Olan \\
& Zaira Navas \\
& William Espino \\
& Rolando Majano \\
& Raúl Alexander Fuentes \\
Amaya Fernández Gordillo \\
Nelson Duarte \\
Nora Mercedes Miranda de López \\
& Óscar Ramón Rosales Meléndez \\
& Vanessa Carolina Martínez \\
& Manuel Antonio Solano \\
\hline &
\end{tabular}




\begin{tabular}{|c|c|}
\hline Organismos internacionales & $\begin{array}{l}\text { Elena Zúñiga } \\
\text { Leonor Calderón } \\
\text { Gordon Jonathan Lewis } \\
\text { Jorge Sagastume } \\
\text { Vilma Núñez } \\
\text { Alicia Navalon } \\
\text { Gilda María Bolt G. (Embajador de Nicaragua) } \\
\text { Xenia Díaz } \\
\text { Miguel Huezo Mixco } \\
\text { Haydee Beatriz Cartagena de Leiva }\end{array}$ \\
\hline Organizaciones no gubernamentales extranjeras & $\begin{array}{l}\text { Presbitero Alejandro Solalinde Guerra } \\
\text { Presbítero Heymann Vásquez } \\
\text { Presbítero Mauro Verzeletti } \\
\text { Fray Tomás González Castillo } \\
\text { Álvaro Caballeros } \\
\text { Rvdo. Phil Anderson } \\
\text { Ricardo Gambetta } \\
\text { Ludin de Chávez } \\
\text { Erika Rusconi } \\
\text { José Mario Ramírez }\end{array}$ \\
\hline Organizaciones no gubernamentales salvadoreñas & $\begin{array}{l}\text { Kiriam Melany Nuila Bonilla } \\
\text { Enrique Merlos } \\
\text { César Ríos } \\
\text { Lucy de Acevedo } \\
\text { Vinicio Sandoval } \\
\text { Verónica Ardón } \\
\text { Miguel Arévalo } \\
\text { Alejandro Benítez Vásquez } \\
\text { Vilma Juárez } \\
\text { Anita Zelaya Galán } \\
\text { Roberto Rubio-Fabián } \\
\text { Alicia Chávez } \\
\text { Luis Alberto López Martínez }\end{array}$ \\
\hline Académicas y académicos & $\begin{array}{l}\text { Miguel Moctezuma Longoria } \\
\text { Jaime Rivas Castillo } \\
\text { María Candelaria Navas } \\
\text { Virginia Quintana } \\
\text { Elsa Ramos } \\
\text { Hazel Jazmín Bolaños Vásquez } \\
\text { Ramón Rivas } \\
\text { Marlon Escamilla } \\
\text { Camila Calles } \\
\text { Gilma Pérez } \\
\text { Jorge Colorado } \\
\text { Teófilo Altamirano Rúa } \\
\text { Carlos Alberto Carcach }\end{array}$ \\
\hline
\end{tabular}




\begin{tabular}{|l|l|}
\hline Periodistas & Mauricio Cáceres \\
& Elena Salamanca \\
& Óscar Díaz \\
& Leonel Herrera \\
& Angélica Cárcamo \\
& Roger Lindo \\
\hline
\end{tabular}

Fuente: Cuadro propio realizado a partir de los programas de la Semana del Migrante realizada en la Utec.

\section{Algunas temáticas desarrolladas}

\begin{tabular}{|c|c|}
\hline \multirow{17}{*}{ Migración y derechos humanos } & Un sueño desaparecido: historias contadas por los(as) hijos(as) de los desaparecidos \\
\hline & Plataforma mínima de los derechos de las personas migrantes \\
\hline & $\begin{array}{l}\text { Abusos y muertos en las fronteras de los migrantes indocumentados en camino a los } \\
\text { Estados Unidos }\end{array}$ \\
\hline & $\begin{array}{l}\text { Crónica de los avatares de los migrantes centroamericanos en tránsito en la frontera sur } \\
\text { de México }\end{array}$ \\
\hline & Informes sobre violación de derechos a deportados \\
\hline & Migrantes centroamericanos en Arriaga \\
\hline & Migrantes centroamericanos en Ixtepec \\
\hline & Migrantes... El costo de las remesas \\
\hline & Acnur y los nuevos retos de las migraciones \\
\hline & $\begin{array}{l}\text { Situación de los derechos humanos de las personas migrantes en la frontera sur de } \\
\text { México }\end{array}$ \\
\hline & Asistencia humanitaria a migrantes en tránsito \\
\hline & Derechos humanos de los migrantes \\
\hline & Migración y derechos laborales en Centroamérica. Análisis jurídico \\
\hline & Desplazamiento generado por la violencia y el crimen organizado \\
\hline & Foro: "Protección de personas migrantes y desplazados" \\
\hline & Derechos humanos de los niños y niñas \\
\hline & $\begin{array}{l}\text { Quehacer de la PDDH con relación a los derechos humanos de las personas migrantes } \\
\text { salvadoreñas }\end{array}$ \\
\hline \multirow{5}{*}{ Migración e historia } & Una mirada histórica y antropológica a las migraciones y el caso de El Salvador \\
\hline & Breve historia de la migración salvadoreña \\
\hline & Las migraciones nahua precolombinas en El Salvador. \\
\hline & $\begin{array}{l}\text { Migraciones nahua-pipiles del postclásico en la cordillera del Bálsamo. El Salvador. } \\
\text { Primera fase }\end{array}$ \\
\hline & Migraciones y diásporas en tiempos prehispánicos \\
\hline
\end{tabular}




\begin{tabular}{|c|c|}
\hline \multirow{4}{*}{ Migración y cultura } & $\begin{array}{l}\text { Sweet home remesas: transformación arquitectónica del oriente del país a partir de la } \\
\text { recepción de remesas familiares }\end{array}$ \\
\hline & La problemática del migrante, procesos de adaptación al nuevo medio \\
\hline & La celebración de la "Purísima" en Santa Rosa de Lima \\
\hline & La problemática actual de las niñeras latinoamericanas en EE.UU. \\
\hline \multirow{13}{*}{ Migración y desarrollo } & Las migraciones como oportunidad para el desarrollo \\
\hline & $\begin{array}{l}\text { Experiencias y lecciones aprendidas por actores: locales, translocales y transnacionales. } \\
\text { Comunidades }\end{array}$ \\
\hline & Sociedad para el desarrollo \\
\hline & $\begin{array}{l}\text { La gestión de la migración con enfoque de desarrollo local: experiencia piloto del } \\
\text { Viceministerio para los Salvadoreños en el Exterior }\end{array}$ \\
\hline & La dinámica de las migraciones y la fuga de cerebros. Una perspectiva centroamericana \\
\hline & Migración y desarrollo económico territorial \\
\hline & Jóvenes emprendedores \\
\hline & Punto Focal El Salvador. Iniciativa Conjunta Migración y Desarrollo (ICMD) \\
\hline & $\begin{array}{l}\text { Mujeres y jóvenes que impulsan el desarrollo comunitario, la agricultura organizada del } \\
\text { municipio de Comasagua y su comercialización }\end{array}$ \\
\hline & Ventanilla para retornados \\
\hline & Inserción laboral de personas retornadas \\
\hline & Diagnóstico de programa de emprendedores para jóvenes \\
\hline & Programas sociales de inserción para prevención de la migración irregular \\
\hline \multirow{8}{*}{ Migración y remesas } & Las remesas colectivas y el migrante colectivo transnacional \\
\hline & El capital humano de la diáspora más allá de las remesas \\
\hline & Los servicios de Fedecaces a las personas migrantes \\
\hline & El costo de las transferencias de remesas \\
\hline & Fedecaces y las migraciones \\
\hline & La responsabilidad social en las migraciones \\
\hline & La incidencia de las remesas en la economía nacional \\
\hline & La dinámica de las remesas \\
\hline \multirow{6}{*}{$\begin{array}{l}\text { Presentación de investigaciones sobre } \\
\text { migración }\end{array}$} & Presentación de investigaciones sobre la dinámica migratoria. \\
\hline & Comentarios al estudio regional sobre la violencia y las migraciones \\
\hline & Transnacionalización de la sociedad salvadoreña producto de las migraciones \\
\hline & Perfil actual de la persona migrante en El Salvador \\
\hline & Migración forzada por violencia social. Una aproximación teórica actual \\
\hline & Niñas, niños y mujeres en la Ruta del Migrante \\
\hline
\end{tabular}




\begin{tabular}{|c|c|}
\hline \multirow{13}{*}{ Migración y acciones desde el gobierno } & Programas del Viceministerio en Atención a las Comunidades Residentes en el Exterior \\
\hline & Proyecciones del nuevo gobierno para los migrantes (2009) \\
\hline & $\begin{array}{l}\text { Análisis del Viceministerio para los Salvadoreños en el Exterior. Una propuesta de } \\
\text { redefinición y reestructuración }\end{array}$ \\
\hline & Gestión del Viceministerio de los Salvadoreños en el Exterior (2011) \\
\hline & Acciones estratégicas del Ministerio de Relaciones Exteriores en apoyo a los migrantes \\
\hline & Ley de Migración y Extranjería \\
\hline & Acciones del Foprel en migración y desarrollo \\
\hline & Situación de los migrantes salvadoreños en la frontera sur de México \\
\hline & La campaña "Si estás pensando migrar, el primer paso es informarte" \\
\hline & Niños, niñas y adolescentes salvadoreños y sus familias (2014) \\
\hline & Gestión del Viceministerio para los Salvadoreños en el Exterior. Período 2014-2015 \\
\hline & Estadísticas de la migración \\
\hline & Apoyo de las personas migrantes en su lugar de destino \\
\hline \multirow{15}{*}{ Migración: NNA y jóvenes } & Juventud, migración y desarrollo \\
\hline & Los jóvenes y la migración internacional \\
\hline & Juventud, mujer y migración \\
\hline & $\begin{array}{l}\text { Encuentro nacional de los jóvenes sobre migración. Plataforma Nacional de los Jóvenes } \\
\text { sobre migración }\end{array}$ \\
\hline & Migración y perspectiva juvenil \\
\hline & Infanticidio social. La migración de NNA salvadoreños \\
\hline & Los niños ancla \\
\hline & Migración, Juventud Ojos abiertos en El Salvador \\
\hline & Migración, proyecto de vida, mujeres jóvenes en El Salvador \\
\hline & NNA migrantes y su ausencia en la Lepina \\
\hline & Niñez migrante y legislación salvadoreña \\
\hline & Juventud y migración \\
\hline & $\begin{array}{l}\text { Informe sobre la situación de NNA salvadoreños migrantes acompañados y no } \\
\text { acompañados }\end{array}$ \\
\hline & Informe sobre la niñez en El Salvador \\
\hline & La niñez migrante en El Salvador \\
\hline \multirow{7}{*}{ Migración, familia y mujer } & Reflexiones sobre la familia transnacional y los cambios socioculturales \\
\hline & Reflexionar sobre mujer, familia y migración \\
\hline & La familia transnacional salvadoreña: un reto para la investigación \\
\hline & $\begin{array}{l}\text { Reflexiones sobre la familia transnacional y los cambios culturales en el escenario } \\
\text { salvadoreño }\end{array}$ \\
\hline & Sueño americano: un ideal de violencia contra las mujeres trabajadoras migratorias \\
\hline & Migración y sociedad \\
\hline & Género y migración \\
\hline
\end{tabular}




\begin{tabular}{|c|c|}
\hline \multirow{8}{*}{ Migración Sur-Sur } & Legalización de los migrantes nicaragüenses en El Salvador \\
\hline & $\begin{array}{l}\text { La experiencia migratoria de los centroamericanos en Guatemala y la Convención de } \\
1990\end{array}$ \\
\hline & Situación de las mujeres migrantes centroamericanas en Chiapas \\
\hline & La situación de hondureños exiliados en El Salvador \\
\hline & La migración extraregional y sus retos en El Salvador \\
\hline & $\begin{array}{l}\text { Proyecto de la DGME-OIM, Embajada de Nicaragua para el proceso de la regulación de } \\
\text { nicaragüenses en El Salvador }\end{array}$ \\
\hline & Salvadoreños y nicaragüenses en la frontera sur de México \\
\hline & Los derechos laborales no tienen fronteras \\
\hline \multirow{2}{*}{ Migración, leyes y reforma migratoria } & Proceso de reforma migratoria en los EE.UU. \\
\hline & La Ley SB-1070 de Arizona y su impacto \\
\hline \multirow{6}{*}{ Migración y trata de personas } & Aspectos generales de la trata de personas \\
\hline & Proyecto de la trata \\
\hline & El delito de la trata de personas. Desafíos en la prevención, combate y atención al delito \\
\hline & Víctimas de trata de personas en la migración \\
\hline & Diagnóstico de migración y trata de niños \\
\hline & $\begin{array}{l}\text { Migración y trata de personas: factores aprovechados por la delincuencia organizada } \\
\text { transnacional }\end{array}$ \\
\hline \multirow{2}{*}{ Migración y población } & Dinámica sociodemográfica y migraciones a la luz del nuevo censo de población \\
\hline & Oportunidades y retos de la migración internacional \\
\hline \multirow{2}{*}{ Migración y medios de comunicación } & Migrantes como noticia \\
\hline & Conversatorio: "Medios de comunicación y migración" \\
\hline
\end{tabular}

Fuente: Cuadro propio realizado a partir de los programas de la Semana del Migrante realizada en la Utec.

\section{Actividades paralelas}

\begin{tabular}{|c|c|c|}
\hline \multirow{4}{*}{ Exposiciones fotográficas } & Patria Sagrada & $\begin{array}{l}\text { DGCE Viceministerio para los Salvadoreños en } \\
\text { el Exterior }\end{array}$ \\
\hline & Los pipiles & $\begin{array}{l}\text { DGCE Viceministerio para los Salvadoreños en } \\
\text { el Exterior }\end{array}$ \\
\hline & $\begin{array}{l}\text { Oficio y sacrificio. Experiencias de un } \\
\text { periodista }\end{array}$ & $\begin{array}{l}\text { Mauricio Cáceres } \\
\text { MUA }\end{array}$ \\
\hline & La Ruta del Migrante & $\begin{array}{l}\text { Fotografías del fotoperiodista Juan de Dios } \\
\text { García Davish, Fundación Carecen Internacional } \\
\text { El Salvador }\end{array}$ \\
\hline Exposición de pinturas y afiches & Alusivas al fenómeno migratorio & $\begin{array}{l}\text { Diferentes ONG salvadoreñas. Montada por el } \\
\text { MUA }\end{array}$ \\
\hline Ferias & $\begin{array}{l}\text { Exposición y distribución de material } \\
\text { de sensibilización e incidencia sobre } \\
\text { migraciones }\end{array}$ & Varias ONG salvadoreñas \\
\hline
\end{tabular}




\begin{tabular}{|c|c|c|}
\hline \multirow{3}{*}{ Actos culturales } & Danzas de Nicaragua & $\begin{array}{l}\text { Caritas El Salvador/Embajada de Nicaragua en } \\
\text { El Salvador }\end{array}$ \\
\hline & Visita guiada a la Sala de las Migraciones & $\begin{array}{l}\text { Técnico Leonardo Regalado } \\
\text { MUA }\end{array}$ \\
\hline & Teatro, danza y música & $\begin{array}{l}\text { Grupo de teatro y danza de la Casa de la } \\
\text { Juventud Ángel de la Guarda. Popotlán, Apopa }\end{array}$ \\
\hline $\begin{array}{l}\text { Exposición temporal de dibujos de } \\
\text { gran formato }\end{array}$ & "Migrantes" & $\begin{array}{l}\text { Maestro Gilberto Arriaza } \\
\text { MUA }\end{array}$ \\
\hline \multirow[t]{2}{*}{ Cine-foro } & $\begin{array}{l}\text { Película: "Emigrantes con muchos } \\
\text { sueños por cumplir" }\end{array}$ & $\begin{array}{l}\text { Ramón Rivas } \\
\text { Director del MUA }\end{array}$ \\
\hline & Película: "La jaula de oro" & Saúl Campos Investigador \\
\hline
\end{tabular}

Fuente: Cuadro propio realizado a partir de los programas de la Semana del Migrante realizada en la Utec.

Como resultado de la celebración de la Semana del Migrante del 2006 al 2015, se considera, según cálculos aproximados, que cada día asistían como promedio unas 200 o 250 personas, entre estudiantes de la Utec de la cátedra de Realidad Nacional (en donde el tema de las migraciones es obligatorio) y personas invitadas de diferentes sectores de la sociedad civil, lo que indica que en una semana sumaban más de mil asistentes que, en diez años, daría como resultado final más de diez mil personas que recibieron la información transmitida durante los eventos.

\section{Referencias}

OECD (2013). "La migración mundial en cifras". Consultado en: http://www.oecd.org/els/mig/SPANISH.pdf

ONU (2013). Comunicado de prensa de las Naciones Unidas. "Diálogo de Alto Nivel sobre la Migración Internacional y el Desarrollo". Consultado en:

http://www.un.org/es/ga/68/meetings/migration/pdf/ press_el_sept\%202013_spa.pdf

RREE (2011). Cancillería, PNUD y UCA presentan "Mapa de las Migraciones Salvadoreñas". Consultado en:

http://www.rree.gob.sv/index.php?option=com_k2\&vie $\mathrm{W}=$ item\&id=1843: canciller\%C3\%ADa-pnud-y-ucapresentan-\%E2\%80\%9Cmapa-de-las-migraciones-sa Ivadore\%C3\%B1as\%E2\%80\%9D\&tmpl=component\& print=1 